Document downloaded from:

http://hdl.handle.net/10251/140232

This paper must be cited as:

Sabek, J.; Martinez-Perez, P.; García-Rupérez, J. (06-2). Computational binding study of cardiac troponin I antibody towards cardiac versus skeletal troponin I. Computational Biology and Chemistry. 80:147-151. https://doi.org/10.1016/j.compbiolchem.2019.04.002

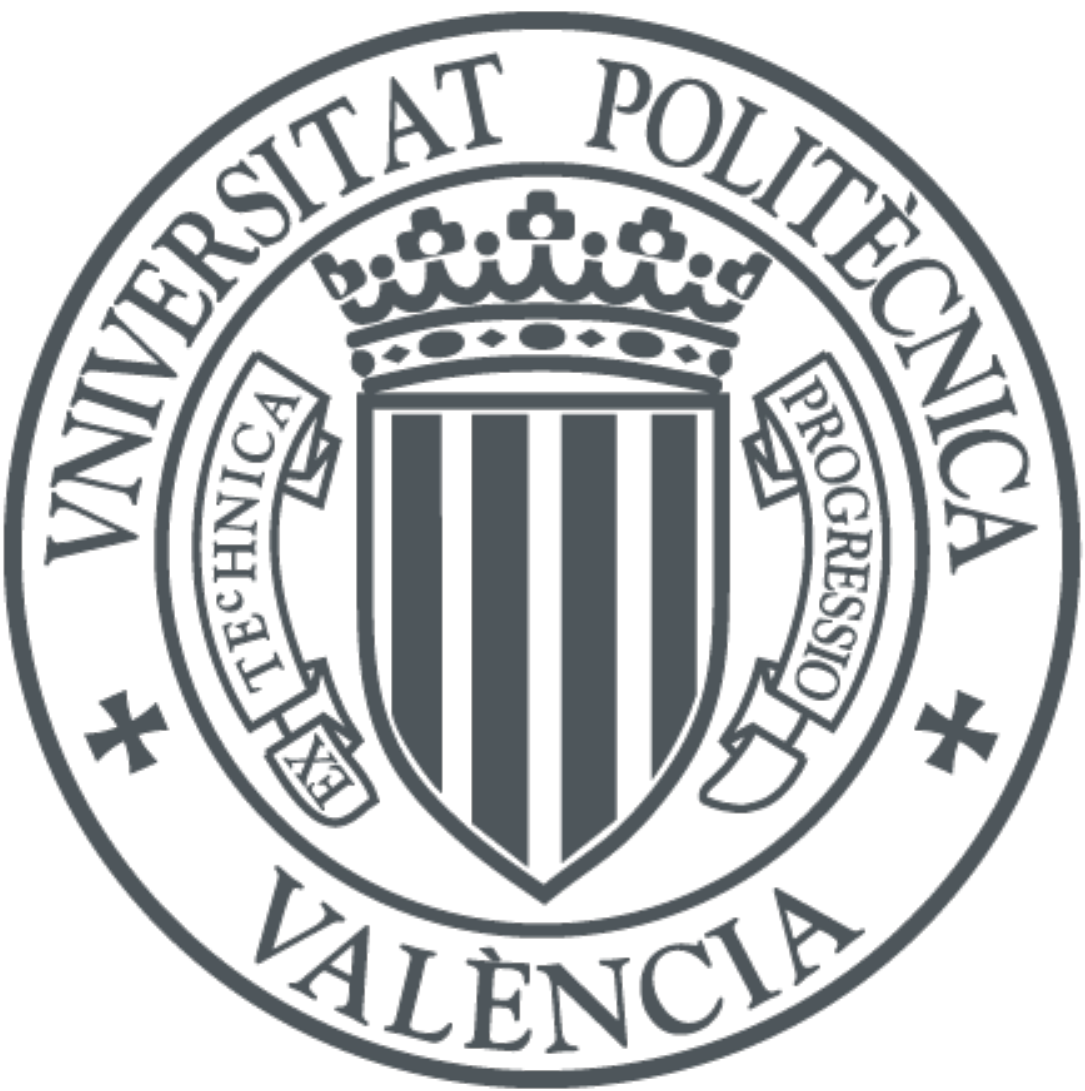

The final publication is available at

https://doi.org/10.1016/j.compbiolchem.2019.04.002

Copyright Elsevier

Additional Information 


\title{
Computational binding study of cardiac troponin I antibody towards cardiac versus skeletal troponin I
}

\author{
Jad Sabek, Paula Martínez-Pérez, Jaime García-Rupérez* \\ Nanophotonics Technology Center, Universitat Politècnica de València, Camí de Vera s/n, Valencia, 46022, Spain \\ *Correspondence: jaigarru@upvnet.upv.es; jasa@ntc.upv.es; Tel.: +34 963877936
}

\begin{abstract}
A computational study of the interaction of cardiac troponin I (cTnI) with its specific antibody and of that antibody with skeletal troponin I (sTnI), the principal interferon of cTnI, is carried out. Computational and simulation tools such as FTSite, FTMap, FTDock and pyDock are used to determine the binding sites of these molecules and to study their interactions and molecular docking performance, allowing us to obtain relevant information related with the antigen-antibody interaction for each of the targets. In the context of the development of immunosensing platforms, this type of computational analysis allows performing a preliminary in-silico affinity study of the available bioreceptors for a better selection when moving to the experimental stage, with the subsequent saving in cost and time.
\end{abstract}

Keywords: Cardiac troponin I (cTnI), skeletal troponin I (sTnI), immunosensing, binding site, molecular docking, FTSite, FTMap, FTDock, pyDock.

\section{Introduction}

Cardiovascular diseases are the first cause of death worldwide and heart failure is the end stage of almost all heart diseases ${ }^{1}$. That heart failure is commonly produced by a damage of the heart myofilaments that can lead to systolic failure, resulting on a decrease of the blood circulation, not sufficing organism needs. One of the most important components of these myofilaments, in terms of its relationship with cardiac damage, is the cardiac troponin (cTn).

With a molecular weight (MW) of approximately $80 \mathrm{kDa}$, cardiac troponin is a complex composed of three protein subunits having a different role in the myocardial contraction ${ }^{2}$. Cardiac troponin T (cTnT; MW 37 kDa) is a tropomyosin-binding subunit that regulates the interaction of the troponin complex with the thin filaments. Cardiac troponin I (cTnI; MW 22 kDa) inhibits the interaction between myosin and actin, responsible of muscle contraction. And finally, cardiac troponin $\mathrm{C}\left(\mathrm{cTnC} ; \mathrm{MW} 18 \mathrm{kDa}\right.$ ) is a $\mathrm{Ca}^{2+}$ binding subunit responsible of regulating the muscle contraction depending on the $\mathrm{Ca}^{2+}$ concentration. The binding of $\mathrm{Ca}^{2+}$ to $\mathrm{cTnC}$ produces a conformational change that reduces the inhibition of $\mathrm{cTnI}$ and thus leads to muscle contraction. That conformational change produced by $\mathrm{cTnC}$ is reversed when $\mathrm{Ca}^{2+}$ levels are restored, thus creating the contraction-relaxation cycle ${ }^{3}$.

When damage of the myofilaments of the cardiac muscle is produced, a breakage of the troponin complex takes place, leading to the release of the troponin subunits (cTnT, cTnI and cTnC) to the blood stream. Thereby, the levels of these cardiac troponin subunits in blood can be used as an indicator of myocardial damage. Note that clinical normal cTnI levels are below $0.11 \mu \mathrm{g} / \mathrm{L}$, more elevated values are considered for severe cardiovascular disease (CVD) patients 4 .

However, troponin is also found in the skeletal muscle, where it is also in charge of controlling the muscle contraction-relaxation cycle ${ }^{5}$. And as for the cTn, skeletal troponin (sTn) subunits (sTnT, sTnI and sTnC) are also released to the blood stream when the myofilaments of the skeletal muscle are damaged. Due to the similarity between cardiac and skeletal troponin, sTn subunits may interfere in the detection of cTn ones, thus limiting the performance of cTn detection-based analysis systems ${ }^{6}$.

Previous works indicate that, from the three cTn subunits, the utility of cTnC for cardiac damage diagnosis is limited by the fact that it presents the same structure than sTnC, with the only difference of the number of $\mathrm{Ca}^{2+}$ binding sites ${ }^{7}$. Regarding TnT and TnI, cTnT presents several specific cardiac isoforms whereas cTnI presents one (in relation with skeletal troponin). Thus, their use in cardiac diagnostic and prognostic applications ${ }^{8}$. Several comparative studies between cTnT cardiac isoforms and cTnI specific cardiac isoform have been carried out, concluding that cTnI exhibits a higher specificity and accuracy than cTnT for the diagnosis of acute myocardial infarction at its early stage ${ }^{9}$. Therefore, cTnI has become the current gold-standard biomarker in clinical diagnosis for the identification of acute 
heart failures. However, it may present a high cross reactivity with the sTnI regarding the interaction with cTnI antibody.

Within this context, in this work we report a computational study of the interaction of a cTnI antibody ( $\alpha \mathrm{cTnI})$ with $\mathrm{cTnI}$ and sTnI. This study allows obtaining information about the biochemical interactions between them and to computationally compare the binding performance and the selectivity of the acTnI towards cTnI versus sTnI rather than experimentally. This information can be relevant for the development of analysis systems for myocardial failure diagnosis based on cTnI detection. First, FTSite and FTMap were used to analyze the structure of the targets (cTnI and sTnI) and the antibody binding fragment (Fab). After the consideration of several possible conformations for cTnI and sTnI (more than a hundred), their most stable conformations (i.e., with the lowest total energy) were used to determine and characterize the binding regions of these molecules. Next, FTDock and pyDock were used to study the molecular docking performance of the cTnI- $\alpha \mathrm{cTnI}$ and sTnI- $\alpha \mathrm{cTnI}$ complexes in order to determine their most stable predicted conformations. Energies and interactions of these predicted conformations allowed us to determine the affinity for each of these complexes and thus to characterize the selectivity.

\section{Materials and methods}

\subsection{Materials}

\subsubsection{Molecules structures}

The X-ray structures of the molecules used in the study were obtained from the Research Collaboratory for Structural Bioinformatics (RCSB) protein data bank (pdb). The IDs of the sTnI and the $\alpha \mathrm{cTnI}$ structures are 1VDI and $4 \mathrm{P} 48$, respectively. For the case of the cTnI, its sequence was extracted from the whole cardiac troponin complex provided by RCSB with the ID 4Y99. Before their use, those molecules were purified by removing those atoms not corresponding with the molecules of interest from the original pdb files and that might be present in the downloaded models (water and ions).

\subsubsection{FTSite and FTMap}

The binding sites of proteins contain the so-called hot spots, specific regions that provide major contributions to the binding free energy. These hot spots are constituted by one or several amino acids that are more likely to bind small molecule probes and compounds with high affinity, and hence are the prime targets in several fields such as immunology or drugs design ${ }^{10}$. Experimental techniques to determine these interactions occurring within the binding regions, e.g., nuclear magnetic resonance or X-ray crystallography, are highly expensive, time consuming and can be limited by the physical constraints of the protein-solvent system ${ }^{11}$. Within this context, the use of computational methods allows characterizing these binding regions numerically rather than experimentally in order to predict their probability of recognition and binding with other molecules ${ }^{12}$.

In order to determine and characterize the binding sites of the cTnI, sTnI and $\alpha \mathrm{cTnI}$ molecules, FTSite and FTMap computing tools were used. These tools perform a statistical search of the entire molecule surface using a Fast Fourier Transform (FFT) based correlation approach in order to determine those regions prickly to bind other probe molecules ${ }^{13}$. FTSite search is based on the fact that the binding sites of a protein generally include several amino acids sequences that are close enough to be reached by a ligand. FTSite characterizes different properties such as volume, hydrophobicity, hydrogen bonding, energy potential, solvent accessibility, desolvation energy and residue propensity. On the other hand, FTMap identifies and map the potential interactions from the binding sites of the protein by analyzing the binding to clusters of 16 organic probes (ethanol, isopropanol, isobutanol, acetone, acetaldehyde, dimethyl ether, cyclohexane, ethane, acetonitrile, urea, methylamine, phenol, benzaldehyde, benzene, acetamide and $\mathrm{N}, \mathrm{N}$-dimethylformamide). FTMap allows representing the obtained energy expression on a grid, which includes attractive and repulsive Van der Waals terms, electrostatic interaction energies and desolvation energies based on Poisson-Boltzmann calculations, resulting in a very accurate way to identify low-energy spot clusters ${ }^{14}$.

\subsubsection{FTDock and pyDock}

Molecular docking is a prediction methodology used to determine the preferred orientation of two molecules when bound to each other to form a stable complex. This preferred orientation can be used to predict their binding affinity, i.e., the association strength between those two molecules. FTDock and pyDock were used to model the biomolecular docking for the cTnI- $\alpha \mathrm{cTnI}$ and sTnI- $\alpha \mathrm{cTnI}$ complexes. Both computational tools perform a rigid-body 
docking of two biomolecules in order to predict their specific binding geometries and their probability/stability given by their lowest total energy (Cheng et al., 2007). Predicted docked complexes were outputted in pdb files, which were displayed using PyMOL 3D viewer. FTDock and pyDock implement different molecular docking algorithms, what was used to confirm that the obtained results were valid. Finally, note that, besides providing the geometric description of the docked complex, pyDock also provides the best docking orientations as scored mainly by electrostatics and desolvation energy ${ }^{17}$.

\subsection{Methods}

The mapping to determine the binding sites was done using FTSite and FTMap, subsequently. Once the binding sites and the amino acids comprising them were determined by FTSite, FTMap was used to map cTnI, sTnI and $\alpha \mathrm{cTnI}$ and determine the interactions of the constitutive amino acids with the test probes. The corresponding pdb files were then uploaded to the FTDock server for the corresponding molecular docking calculations. Several conformations were generated and the most suitable prediction was chosen for the next steps. In parallel, the same process was done using pyDock for a comparative study of the docking results obtained with FTDock. The 100 most suitable conformations were generated in pdb files, only considering the most stable one of each complex. The docking results obtained using both methodologies showed no difference, thus confirming the correctness of the analysis carried out.

\section{Results and discussion}

First, the binding sites on the molecules surfaces were determined for the cTnI, sTnI and $\alpha \mathrm{cTnI}$. Even when the structure of a molecule is determined by crystallography in a complex with a ligand, a complete description of its binding sites with that ligand cannot be determined experimentally because complex structures may not fully exploit the overall properties of the binding site. Moreover, knowledge of the possible binding sites in the structure of a molecule also enables us to analyze and classify them through their binding sites profiles. Figure 1 shows the chosen binding sites for cTnI, sTnI and acTnI determined using FTSite.
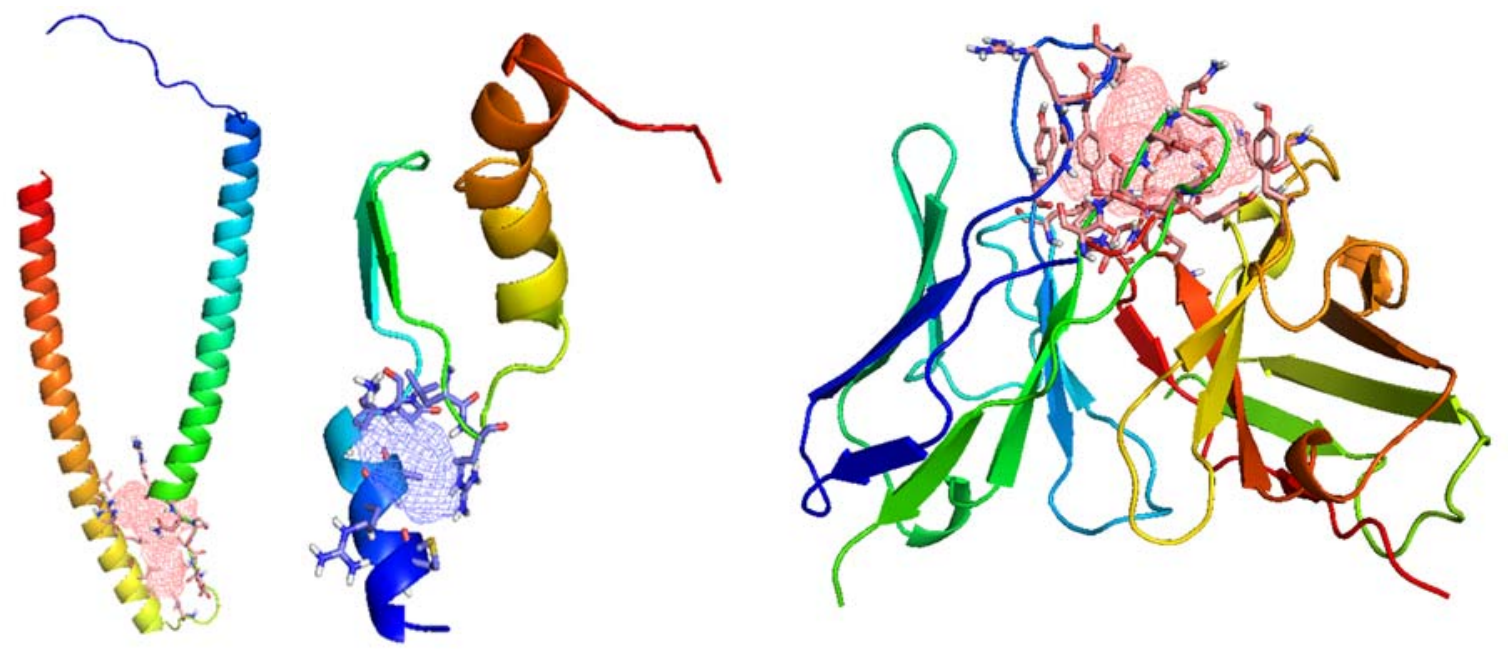

Figure 1. FTSite results showing the binding sites (colored grids) of (left) cTnI, (center) sTnI and (right) Fab region of the $\alpha \mathrm{cTnI}$. The visualization has been done using PyMOL.

FTSite also provides the amino acids sequences forming the binding sites as shown in Table 1. 
Table 1. Complete description of the amino acids sequences forming the binding sites given by FTSite for cTnI, sTnI and Fab region of the $\alpha \mathrm{cTnI}$.

\begin{tabular}{ccc}
\hline cTnI & sTnI & acTnI \\
\hline Leu49; Leu52; & Met134; Arg137; Ala138; & Leu2; Arg25; Tyr26; Tyr27; Asp28; \\
Lys72; Arg79; & Asn139; Leu140; Lys141, & Ala44; Pro45; Thr47; Glu51; Lys54; \\
Ala80; Gln81; & Gln142; Val143; Lys145; Glu149; & Asn58; Asp93; Asp94; Asn95; Asn97; \\
Pro82; Leu83; & Glu151; Lys152; Asp153; & Pro98; Thr99, Phe101; Gly102; Leu130; \\
Glu84; Leu93; & Leu154; Arg155; Asp156; & Leu132; Gly172; Leu173; Glu174; \\
Gln99; Leu100; & Gly158; Asp159; Trp160; & Trp175; Gln180; Gly185; Ala190; \\
Arg103; Val104; & Arg161; Asn163; Glu165; Ser168; & Ala191; Lys226; Asp227; Ser229; \\
Val107; Glu110, & Gly169; Met170; Gly172; & Ser230; Asp231; Tyr234; Asp237; \\
Arg111; Glu115 & Arg173; Lys74; Phe177 & Ser238; Asp240; Trp242; His244 \\
\hline
\end{tabular}

Once the binding sites sequences were obtained using FTSite, FTMap was used to determine the number of Hbond interactions between the amino acids comprising the binding site and the organic probe molecules tested by FTMap. Tables 2, 3 and 4 show the H-bond interactions with the probe molecules for cTnI, sTnI and $\alpha \mathrm{cTnI}$, respectively. The first column lists the amino acid number within the binding site, the second lists the amino acid 3letter code and the third column lists the number of H-bond contacts between the amino acids and the probe molecules.

Table 2. cTnI H-bond interaction results between the amino acids from the binding site and the probe molecules as identified by FTMap.

\begin{tabular}{ccc}
\hline cTnI amino acid number & Amino acid 3-letter code & Number of H-bond contacts \\
\hline $49 ; 52 ; 72 ; 79 ; 80 ; 81 ;$ & Leu; Leu; Lys; Arg; Ala; Gln; & $66 ; 15 ; 30 ; 556 ; 26 ; 1510 ;$ \\
$82 ; 83 ; 84 ; 93 ; 99 ; 100 ;$ & Pro; Leu; Glu; Leu; Gln; Leu; & $174 ; 119 ; 918 ; 490 ; 26 ; 761 ;$ \\
$103 ; 104 ; 107 ; 110 ; 111 ; 115$ & Arg; Val; Val; Glu; Arg; Glu & $531 ; 23 ; 830 ; 431 ; 207 ; 20$ \\
\hline
\end{tabular}

Table 3. sTnI H-bond interaction results between the amino acids from the binding site and the probe molecules as identified by FTMap.

\begin{tabular}{ccc}
\hline sTnI amino acid number & Amino acid 3-letter code & Number of H-bond contacts \\
\hline $134 ; 137 ; 138 ; 139 ; 140 ; 141 ;$ & Met; Arg; Ala; Asn; Leu; Lys; & $102 ; 1 ; 5 ; 6 ; 13 ; 133 ;$ \\
$142 ; 143 ; 145 ; 149 ; 151 ; 152 ;$ & Gln; Val; Lys; Glu; Glu; Lys; & $84 ; 63 ; 1094 ; 60 ; 2014 ; 11 ;$ \\
$153 ; 154 ; 155 ; 156 ; 158 ; 159 ;$ & Asp; Leu; Arg; Asp; Gly; Asp; & $3708 ; 2 ; 883 ; 2 ; 3 ; 3 ;$ \\
$160 ; 161 ; 163 ; 165 ; 168 ; 169 ;$ & Trp; Arg; Asn; Glu; Ser; Gly; & $135 ; 165 ; 5 ; 738 ; 1041 ; 981 ;$ \\
$170 ; 172 ; 173 ; 174 ; 177$ & Met; Gly; Arg; Lys; Phe & $1020 ; 3 ; 618 ; 76 ; 243$ \\
\hline
\end{tabular}

Table 4. $\alpha \mathrm{cTnI}$ H-bond interaction results between the amino acids from the binding site and the probe molecules as identified by FTMap.

\begin{tabular}{ccc}
\hline $\boldsymbol{\alpha c T}$ I amino acid number & Amino acid 3-letter code & Number of H-bond contacts \\
\hline $2 ; 25 ; 26 ; 27 ; 28 ; 44 ;$ & Leu; Arg; Tyr; Tyr; Asp; Ala; & $19 ; 151 ; 233 ; 517 ; 1 ; 2 ;$ \\
$45 ; 47 ; 51 ; 54 ; 58 ; 93 ;$ & Pro; Thr; Glu; Lys; Asn; Asp; & $693 ; 2676 ; 233 ; 11 ; 369 ; 7 ;$ \\
$94 ; 95 ; 97 ; 98 ; 99 ; 101 ;$ & Asp; Asn; Asn; Pro; Thr; Phe; & $282 ; 48 ; 108 ; 338 ; 401 ; 1804 ;$ \\
$102 ; 130 ; 132 ; 172 ; 173 ; 174 ;$ & Gly; Leu; Leu; Gly; Leu; Glu; & $171 ; 43 ; 215 ; 33 ; 646 ; 39 ;$ \\
$175 ; 180 ; 185 ; 190 ; 191 ; 226 ;$ & Trp; Gln; Gly; Ala; Ala; Lys; & $2220 ; 395 ; 14 ; 923 ; 66 ; 24 ;$ \\
$227 ; 229 ; 230 ; 231 ; 234 ; 237 ;$ & Asp; Ser; Ser; Asp; Tyr; Asp; & $6 ; 1165 ; 48 ; 82 ; 1392 ; 816 ;$ \\
$238 ; 240 ; 242 ; 244$ & Ser; Asp; Trp; His & $727 ; 1052 ; 2228 ; 8$ \\
\hline
\end{tabular}

After the identification and mapping of binding sites and the interactions of their constitutive amino acids sequences, the next step is studying the molecular docking performance for the cTnI and sTnI to $\alpha \mathrm{cTnI}$ binding. This process of docking provides the specific interaction between the proteins and the antibody Fab region taking into account spatial distribution, Van der Waals interactions and rotational restrictions, among others. For the docking analysis, FTDock and pyDock were used. FTDock generates several output pdb files with docking probabilities organized from the highest to the lowest probable one. On the other hand, pyDock generates several pdb files of the docked complexes being randomly organized, but results are accompanied by other generated files describing 
energies, positions and angles that can be used to organize the predicted complexes. The ranking of molecular docking complexes obtained with both methods are the same, thus confirming the docking results. Figure 2 shows the docked complexes obtained for cTnI- $\alpha \mathrm{cTnI}$ and sTnI- $\alpha \mathrm{cTnI}$.
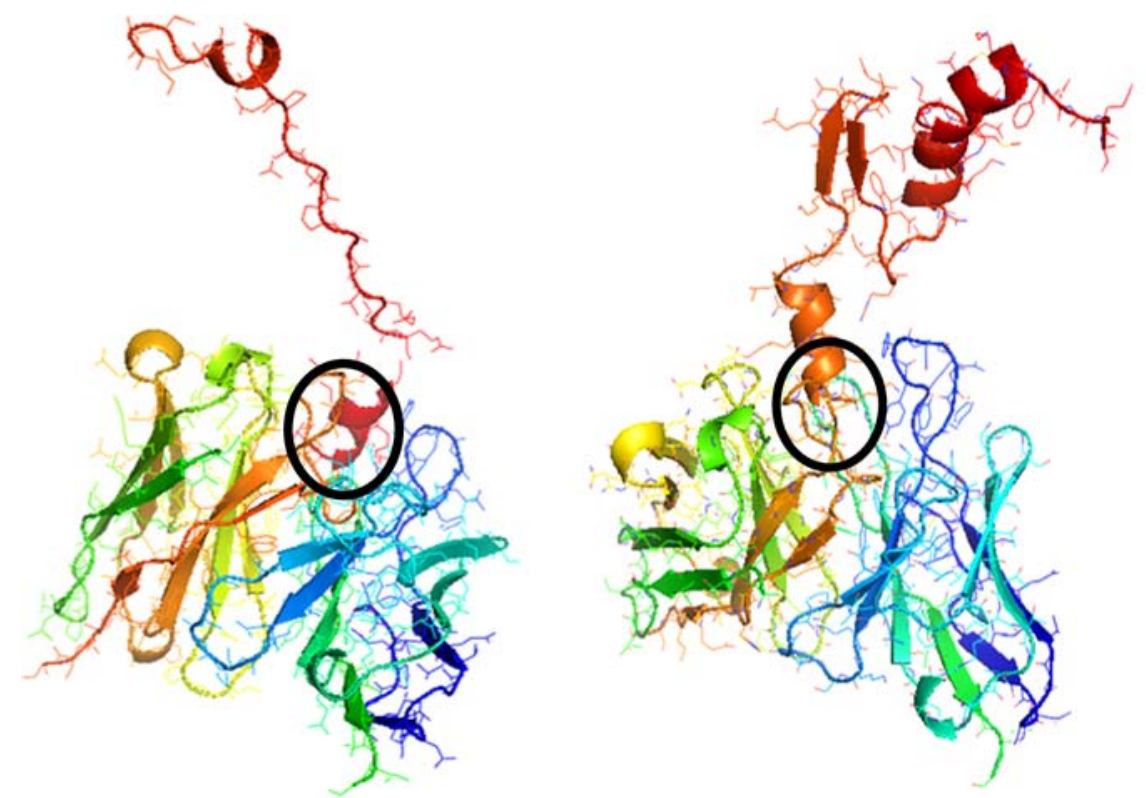

Figure 2. Most probable docked configurations of (left) cTnI- $\alpha c T n I$ and (right) sTnI- $\alpha c T n I$ complexes. Binding zones are highlighted with black circles.

Table 5 shows the identified H-bond interactions for the cTnI- $\alpha \mathrm{cTnI}$ and the sTnI- $\alpha \mathrm{c} T n I$ complexes obtained using FTDock and pyDock. Three H-bonds are predicted for both complexes. However, while these H-bonds are formed by three different amino acids for the cTnI- $\alpha \mathrm{cTnI}$ complex, for the sTnI- $\alpha \mathrm{cTnI}$ complex two of them are formed by the same amino acid from the sTnI (Glu149), what is translated into a weaker H-bond than for cTnI- $\alpha \mathrm{cTnI}$. This fact determines the higher affinity of acTnI towards cTnI than for sTnI.

Table 5. H-bonds for the cTnI- $\alpha \mathrm{cTnI}$ and the sTnI- $\alpha \mathrm{cTnI}$ complexes identified using FTDock and pyDock.

\begin{tabular}{ccc}
\hline & cTnI-acTnT & sTnI-acTnT \\
\hline \multirow{3}{*}{ H-Bonds } & Gln81-Phe101 & Lys145-Lys54 \\
& Glu84-Ser229 & Glu149-Thr47 \\
& Leu100-Trp242 & Glu149-Trp242 \\
\hline
\end{tabular}

Having identified the most stable predicted structures, the formed cTnI- $\alpha \mathrm{cTnI}$ and sTnI- $\alpha \mathrm{cTnI}$ complexes were visualized using PyMOL to depict the specific interactions between the amino acids sequences previously identified (see Fig. 3). 

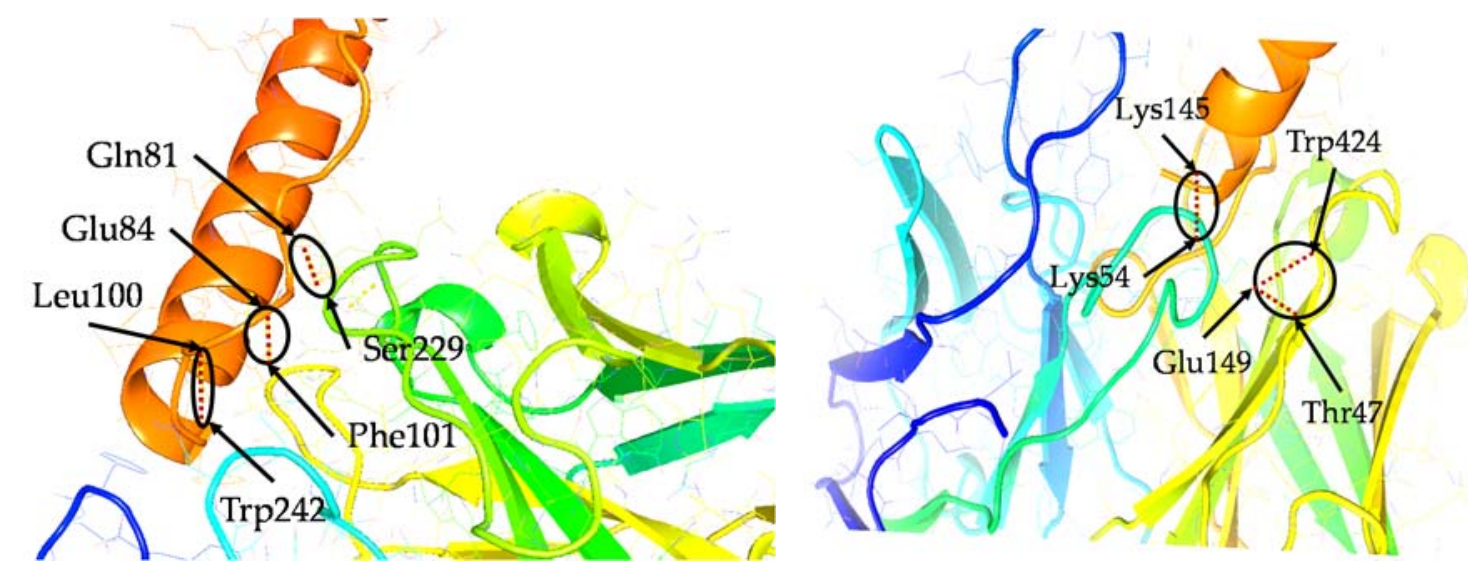

Figure 3. Detailed 3D view of the (left) cTnI- $\alpha \mathrm{cTnI}$ and (right) sTnI- $\alpha \mathrm{cTnI}$ docked complexes. H-bonds between the amino acids from the binding sites are depicted with dashed red lines and highlighted with black circles.

FTDock provides more than a thousand possible combinations for the two docked troponins to acTnI. Different parallel data were also given to distinguish the most stable configurations as previously mentioned. Table 6 depict the parameters describing energies of the most stable docking configurations for the cTnI- $\alpha \mathrm{cTnI}$ and sTnI- $\alpha \mathrm{cTnI}$ complexes. We can see that the total energy of the cTnI- $\alpha \mathrm{cTnI}$ complex is considerably lower than for the sTnI- $\alpha \mathrm{cTnI}$ complex, what indicates a higher affinity of $\alpha \mathrm{cTnI}$ towards cTnI than for sTnI. This confirms the lower affinity for the sTnI- $\alpha \mathrm{cTnI}$ complex predicted from the H-bonds performance previously shown in Table 5 and Fig. 3.

Table 6. Energy data $(\mathrm{Kcal} / \mathrm{mol})$ for the most stable docked complexes for cTnI- $\alpha \mathrm{cTnI}$ and sTnI- $\alpha \mathrm{cTnI}$.

\begin{tabular}{ccccc}
\hline \multirow{2}{*}{ Complexes } & $\begin{array}{c}\text { Electrostatic } \\
\text { interaction }\end{array}$ & Desolvation & $\begin{array}{c}\text { Van der } \\
\text { Waals }\end{array}$ & Total \\
\hline cTnI- $\alpha$ cTnI & -11.866 & -20.190 & 28.475 & $\mathbf{- 2 9 . 2 0 8}$ \\
sTnI- $\alpha \mathrm{cTnI}$ & -29.635 & -3.683 & 72.809 & $\mathbf{- 2 6 . 0 3 7}$ \\
\hline
\end{tabular}

Therefore, this study reveals that the selected $\alpha \mathrm{cTnI}$ antibody is suitable for the experimental development of a cTnI-selective biosensing system in a subsequent stage. By performing this type of theoretical and computational analysis, we are able to preliminary determine the suitability of a certain capture antibody without the need of investing a large amount of resources on its experimental selection as well as to obtain more interesting information about it. This allows defining a better initial design of our biosensing experiments, with an enormous thrift on cost, effort and time.

\section{Conclusions}

In this work, we have demonstrated that an affinity study can be performed computationally rather than experimentally, what can dramatically reduce time and cost when developing immunosensing systems. As far as we know, this is the first time that this type of computational affinity study is performed for cardiac troponin I. The obtained computational affinity and selectivity results are highly important, since they compare the suitability of cTnI and its principal interferon $\mathrm{sTnI}$ to bind $\alpha \mathrm{cTnI}$ for a cross reactivity study, with the aim of a correct selection of a bioreceptor for the development of a cTnI biosensor for an effective, selective, fast and direct early detection of a myocardial failure.

Acknowledgments: This work was supported by the Horizon 2020 Programme of the European Union under the project H2020PHC-634013 (PHOCNOSIS).

We highly acknowledge Barcelona supercomputing center, universitat politècnica de catalunya for their technical support.

Conflicts of Interest: The authors declare no conflict of interest. 


\section{References}

1. Pagidipati, N. J. \& Gaziano, T. A. Estimating deaths from cardiovascular disease: a review of global methodologies of mortality measurement. Circulation 127, 749-56 (2013).

2. Gomes, A. V., Potter, J. D. \& Szczesna-Cordary, D. The Role of Troponins in Muscle Contraction. IUBMB Life (International Union Biochem. Mol. Biol. Life) 54, 323-333 (2002).

3. Collinson, P., Boa, F. G. \& Gaze, D. C. Measurement of cardiac troponins. Review Article Ann Clin Biochem 38, (2001).

4. Sagarad, S. V, Singh Thakur, B., Reddy, Balasubramanya, S. S., Joshi, M. \& Kerure, S. B. Elevated Cardiac Troponin (cTnI) Levels Correlate with the Clinical and Echocardio-graphic Evidences of Severe Myocarditis in Scorpion Sting Envenomation. J. Clin. Diagnostic Res. 6, 1369-1371 (2012).

5. Gordon, A. M., Homsher, E. \& Regnier, M. Regulation of Contraction in Striated Muscle. Physiol. Rev. 80, 853-924 (2000).

6. Eriksson, S. et al. Negative interference in cardiac troponin I immunoassays from a frequently occurring serum and plasma component. Clin. Chem. 49, 1095-104 (2003).

7. Yang, Z., Yamazaki, M., Shen, Q. W. \& Swartz, D. R. Differences between Cardiac and Skeletal Troponin Interaction with the Thin Filament Probed by Troponin Exchange in Skeletal Myofibrils. Biophys. J. 97, 183194 (2009).

8. Collinson, P. O., Gaze, D. \& Goodacre, S. The clinical and diagnostic performance characteristics of the high sensitivity Abbott cardiac troponin I assay. Clin. Biochem. 48, 275-281 (2015).

9. Apple, F. S., Ler, R. \& Murakami, M. M. Determination of 19 cardiac troponin I and T assay 99th percentile values from a common presumably healthy population. Clin. Chem. 58, 1574-81 (2012).

10. Yuan, Y., Pei, J. \& Lai, L. Binding Site Detection and Druggability Prediction of Protein Targets for StructureBased Drug Design. Curr. Pharm. Des. 19, 2326-2333 (2013).

11. Allen, K. N. et al. An Experimental Approach to Mapping the Binding Surfaces of Crystalline Proteins ${ }^{\dagger} . J$. Phys. Chem. 100, 2605-2611 (1996).

12. Katchalski-Katzir, E. et al. Molecular surface recognition: determination of geometric fit between proteins and their ligands by correlation techniques. Proc. Natl. Acad. Sci. U. S. A. 89, 2195-9 (1992).

13. Dennis, S., Kortvelyesi, T. \& Vajda, S. Computational mapping identifies the binding sites of organic solvents on proteins. Proc. Natl. Acad. Sci. U. S. A. 99, 4290-5 (2002).

14. Kozakov, D. et al. The FTMap family of web servers for determining and characterizing ligand-binding hot spots of proteins. Nat. Protoc. 10, 733-755 (2015).

15. Brenke, R. et al. Fragment-based identification of druggable 'hot spots' of proteins using Fourier domain correlation techniques. Bioinformatics 25, 621-627 (2009).

16. Cheng, T. M.-K., Blundell, T. L. \& Fernandez-Recio, J. pyDock: Electrostatics and desolvation for effective scoring of rigid-body protein-protein docking. Proteins Struct. Funct. Bioinforma. 68, 503-515 (2007).

17. Jiménez-García, B., Pons, C. \& Fernández-Recio, J. pyDockWEB: a web server for rigid-body protein-protein docking using electrostatics and desolvation scoring. Bioinformatics 29, 1698-1699 (2013). 